Case study

\title{
LARGE PRIMARY TUBERCULOUS PSOAS ABSCESS IN AN HIV PATIENT - A CASE REPORT
}

\author{
Rakesh TP1', Kidangazhiathmana A', Jyothish K', Amrutha PC ${ }^{1}$, Satheeshbabu TV², \\ ${ }^{1}$ Government Medical College, Thrissur, Kerala, India \\ 2 Malabar Cancer Center, Thalassery, Kerala, India
}

\begin{abstract}
Tuberculosis (TB) remain as one of the leading opportunistic infection in patients with Human immunodefficiency virus (HIV) infection in developing countries. Here we report a case of huge primary psoas abscess of tubercular origin in an HIV patient who presented with hip pain and limping. Psoas sign was evident on examination and CT scan of abdomen revealed a large iliopsoas abscess extending from abdomen to upper part of thigh. Patient underwent percutaneous drainage of abscess and improved with anti-tuberculous drugs while continuing anti-retroviral therapy.
\end{abstract}

Key words: HIV, AIDS-Related Opportunistic Infections, Psoas Abscess, Tuberculosis

\section{INTRODUCTION}

Human immunodeficiency virus (HIV) and Tuberculosis (TB) are the first and second most cause of death due to an infectious disease and in 2012, 0.3 million HIV-associated TB deaths were reported worldwide. ${ }^{1}$ HIV and TB accelerate their course each other increasing the mortality and morbidity rates. Unusual presentations of TB due to altered immune response pose diagnostic challenge in HIV. Clinical manifestations due to the underlying TB depends on the CD4+ count- patients with higher counts may present with cavitatory upper lobe lung lesions or caseating lymph nodes and those with lower counts develop more disseminated disease like military TB. Here is an account of a patient with HIV on highly active antiretroviral therapy (HAART) and developed relapse of TB after two years in the form of huge iliopsoas abscess. The case is presented considering the

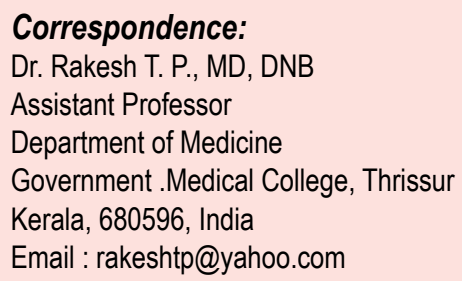

sheer size of the abscess, recovery with treatment and also to remind the possibility of psoas abscess in HIV patients with hip pain.

\section{Case report}

32 year old male, HIV-1 positive, presented with history of left hip pain and limp on walking for 3 weeks. On examination, he was afebrile and vital signs were normal. There was no pallor, lymphadenopathy or oral candidiasis. Chest was clear to auscultation and abdomen was soft without any palpable mass. His left limb was kept in a partial hip flexion and was painful on forced extension (Psoas sign, figure 1). Patient was on Zidovudine $300 \mathrm{mg}$, Lamivudine $150 \mathrm{mg}$, and Nevirapine 200 $\mathrm{mg}$; all tablets twice daily for the last 2 years with improving CD4+ count. He was treated for TB involving the intra-abdominal lymph nodes two years back and at that time CD4+ count was $80 /$ $\mathrm{mm}^{3}\left(500-1300 / \mathrm{mm}^{3}\right)$. This time CD4+ count was 270/mm3 (Viral load was not done).CT scan of abdomen with contrast revealed a large lobulated thin walled fluid collection in the left iliopsoas compartment from the level of disc between first and second lumbar vertebrae to about six $\mathrm{cm}$ below the left lesser trochanter (figure 2 and 3). Nearby vertebrae and disc spaces were intact. Chest $X$ ray was normal. Abscess was sampled percutaneously 
through a needle and yielded yellowish caseating material which stained negative for acid fast or gram positive bacilli. In view of caseating nature of the aspirated material, absence of any other bacilli in gram stain and radiological features, a diagnosis of primary tubercular psoas abscess was made. The abscess was approached.

percutaneously through a small incision posteriorly just above the left iliac crest and about $500 \mathrm{ml}$ of pus was drained. He was put on WHO Category II anti-tuberculous regimen. HAART was continued; Nevirapine was replaced with Efavirenz $600 \mathrm{mg}$ once daily. Patient improved over a period of 4 months and at one year he is very well ambulant with a small limp.

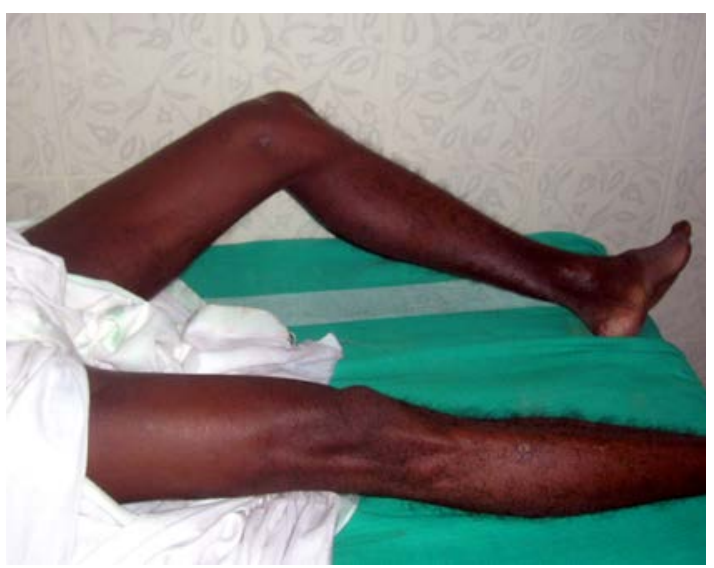

Figure 1. Left hip kept in a semi flexed position due to painful spasm of psoas muscle

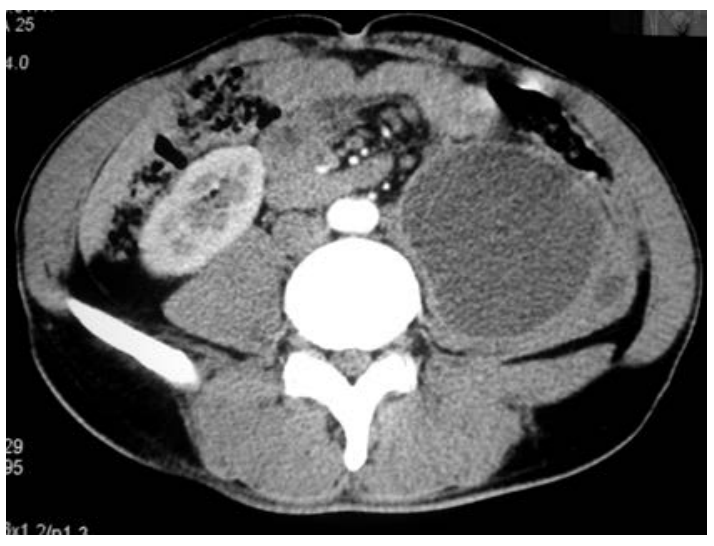

Figure 2. CT scan showing the huge abscess of the left iliopsoas muscle

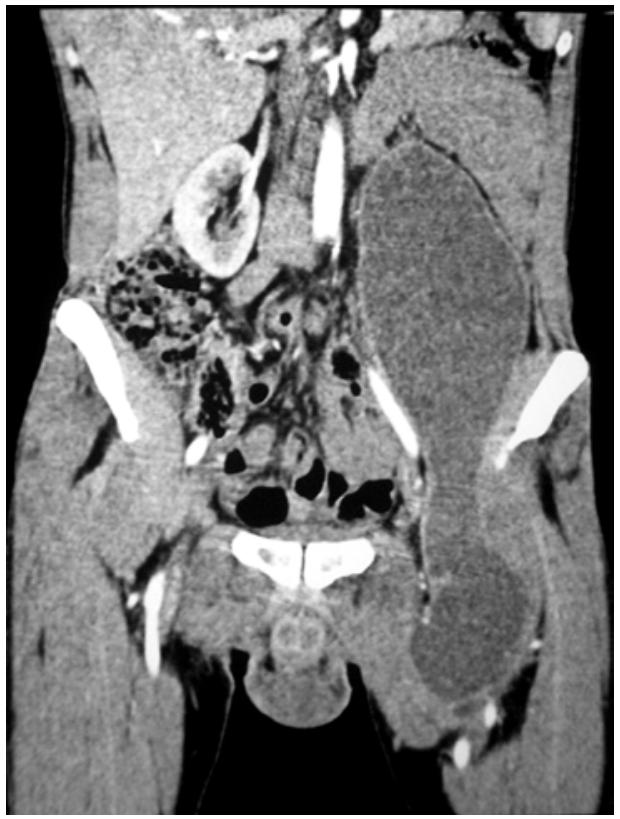

Figure 3. CT scan showing the huge abscess of the left iliopsoas muscle

\section{DISCUSSION}

Psoas and iliacus muscle-together called as iliopsoas- are located in the iliopsoas compartment and originate form the lateral aspects of $12^{\text {th }}$ thoracic to $5^{\text {th }}$ lumbar vertebrae, to be inserted to the lesser trochanter of femur. Infections can enter into this muscle form it's neighboring structures like iliac lymph nodes, sigmoid colon and abdominal aorta.

Psoas abscess in immune competent patients is either primary or secondary due to peri-nepheric abscess, lumbar discitis or infected abdominal aortic aneurysm. ${ }^{2}$ Staphylococcus aureus remain as the most common aetiological agent and others are Escherichia coli, Klebseilla pneumonia, Enterobacter and Mycobacterium tuberculosis. ${ }^{3}$

Most of the time, the tuberculous psoas abscess is secondary; source of infection being osteomyelitis of the spine. ${ }^{3}$ The primary psoas abscess is rare especially in HIV patients with only few case reports. ${ }^{4}$ 
The Psoas abscess may present with pain involving back, hip or lower abdomen. Patient can develop a limp with pain radiating to back of thigh. Fever will be present in majority and psoas abscess remains as one of the causes for fever of unknown originin a case series, median time between symptom onset and diagnosis was $>42$ days in one third of the patients. ${ }^{5}$

Diagnosis is established with MRI or a contrast enhanced CT scan of abdomen as ultrasound is a less sensitive method. Sampling of the material can be done with needle aspiration. Though classical treatment remain as open surgical drainage ${ }^{6}$, laproscopic ${ }^{7}$ and percutaneous drainage are also described with successful outcome. ${ }^{8}$ Most of them recover but sequel can occur due to osteomyelitis of adjacent bones and scarring or weakness of muscle. ${ }^{9}$

Patient in our case had abdominal lymph node TB during the period of lower CD4+ count (before initiating HAART). After two years of HAART, TB relapse took the form of caseating mass, most likely due to higher CD4+ count (Immune reconstitution). The case is presented due to several unique features- patient presented with hip pain rather than fever, it was a primary abscess and had a good outcome with open drainage and anti-tuberculous drugs.

\section{CONCLUSIONS}

With the advent of HIV, the psoas abscess due to $T B$ is expected to increase in developing countries. The diagnosis, especially in earlier stages can be challenging as blunted immunological response may not produce noticeable signs of infection in HIV patients. Primary tuberculous psoas abscess in HIV patients remain rare and there there are no definite guidelines regarding the treatment as there are only a very few case reports. In patients with HIV, the possibility of this entity should be kept in mind in cases of persistent hip pain.

\section{REFERENCES}

1. WHO Global Tuberculosis Report 2013.

2. Alvi AR, Ur Rehman Z, Nabi ZURG. Pyogenic psoas abscess: case series and literature review. Trop Doct. 2010;40:56-8.

3. Van den Berge M, de Marie S, Kuipers T, Jansz AR, Bravenboer B. Psoas abscess: report of a series and review of the literature. Neth $\mathrm{J}$ Med. 2005;63:413-6.

4. Assenza M, Antoniozzi A, Clementi I, Bartolucci $P$, Ciccarone $F$, Simonelli $L$, et al. Primary psoas abscess in a patient affected by acquired immunodeficiency syndrome: a rare case according to the reviewed literature. Clin Ter. 159:261-3.

5. Navarro López V, Ramos JM, Meseguer V, Pérez Arellano JL, Serrano R, García Ordóñez MA, et al. Microbiology and outcome of iliopsoas abscess in 124 patients. Medicine. 2009;88:120-30.

6. MacGillivray DC, Valentine RJ, Johnson JA. Strategies in the management of pyogenic psoas abscesses. Am Surg. 1991;57:701-5.

7. Atkin $\mathrm{G}$, Qurashi K, Isla A. Laparoscopic drainage of bilateral tuberculous psoas abscesses. Surg Laparosc Endosc Percutan Tech. 2005;15:380-2.

8. Horváth G, BodaA, Repal. CT-guided percutaneous drainage in the management of psoas abscesses. Orv Hetil. 1994 Nov 20;135:2597-602.

9. Bickels J, Ben-Sira L, Kessler A, Wientroub S. Primary pyomyositis. J Bone Joint Surg Am. 20021;84-A:2277-86. 